\title{
Educar en tiempos de virtualidad. Estrategias educativas
}

\author{
Luisa Rodríguez Zavala \\ Universidad Ricardo Palma, Lima, Perú \\ luisa.rodriguez@urp.edu.pe
}

\begin{abstract}
RESUMEN
El mundo atraviesa una de las mayores crisis sanitarias y económicas de su historia contemporánea producto de la pandemia por la COVID 19 y sus variantes; que entre sus múltiples impactos sociales, ha obligado a casi todos los países a implementar la educación a distancia como el soporte fundamental para continuar con el proceso educativo en todos sus niveles. A partir de esa instancia comienzan a vislumbrarse nuevas estrategias para la intensificación de la Educación a Distancia como un proceso irreversible. En tal sentido la virtualización es parte de la transformación digital, enmarcada en la revolución tecnológica en curso, en la cual el acceso a la educación virtual permitiría el ideal de la educación permanente y la democratización del acceso a la educación. El objetivo del artículo es reflexionar sobre las estrategias educativas necesarias para su instrumentación en la virtualidad, las cuales abren nuevas posibilidades individuales e institucionales para una expansión sin precedentes del aprendizaje en casa.
\end{abstract}

Palabras clave: Estrategia educativas, educación a distancia, virtualidad, tecnologías educativas, aprendizaje

\section{Teaching in virtual times. Teaching strategies}

\begin{abstract}
The world is going through one of the greatest sanitary and economic crises of the contemporary history because of COVID-19 and variants Pandemic. Among its multiple social impacts, it has forced almost all countries to implement remote education as the main support to continue with educational process at all levels. Considering this situation, new strategies to intensify the remote education are appearing as an irreversible process. In this sense, virtuality is part of digital transformation in the frame of current technological revolution in which the access to virtual educational would make real the dream of the permanent education and the democratization of access to education. The purpose of this article is to reflect on the teaching strategies required to the instrumentation in virtuality. These strategies bring new individual and institutional opportunities to the unprecedented expansion of learning at home.
\end{abstract}

KeYwords: Teaching strategies, remote education, virtuality, educational technologies, learning 


\section{Introducción}

A inicios del siglo XXI se ha producido un acelerado proceso de desarrollo de las tec-nologías de la comunicación y el internet, que en sus más recientes fases la multimedia y lo interactivo en el campo educativo abre nuevas posibilidades individuales e institucionales para una expansión sin precedentes del aprendizaje, la educación a distancia se ha virtualizado y su acceso ella se constituye como un derecho educativo fundamental de nuestros tiempos. En este nuevo escenario comienzan a aparecer nuevos actores, métodos y estrategias para organizar la educación a distancia como un derecho digital e inclusivo como un mecanismo para promover mayor igualdad educativa en el país, que se encuentra con enormes brechas digitales y tecnológicas.

En un principio, la enseñanza a distancia se justificó como método sustitutorio: se realizaban a distancia aquellos estudios que no contaban con suficientes recursos para darse de forma presencial. La situación actualmente es distinta, la enseñanza a distancia ha seguido un acelerado proceso de desarrollo en la medida que se fueron logrando grandes avances en los soportes tecnológicos de la comunicación a través del internet y la virtualidad, que sumado a una demanda educativa creciente, permitió la expansión de la educación a distancia y que en el contexto de las condiciones actuales de la cri-sis sanitaria mundial originada por la pandemia, ha dado acelerado e intensificado la educación a distancia como un proceso que ha llegado para instalarse como parte de nuestra realidad educativa de manera permanente. Que a la vez es necesario tomar conciencia que resulta imposible materializar la educación permanente con la permanente escolarización; no solo por motivos económicos sino también técnicos-científicos. En tal sentido la importancia de las Estrategias de Comunicación en la Educación a Distancia hoy juega un rol fundamental y representa una herramienta eficaz de ayudar a hacer realidad el ideal de la educación permanente y la democratización del acceso a una educación que debe ser de alta calidad. El presente artículo está dividido en cuatro partes la primera plantea una descripción de la funcionalidad didáctica de las tecnologías de la educación, la segunda aborda sobre la comunicación en el sistema de educación, la tercera parte es acerca de las estrategias educativas. Finalmente, se plantean algunas conclusiones sobre la relación existente entre las estrategias de comunicación educativa y la potenciación y consolidación de los aprendizajes.

\section{Funcionalidad didáctica de las tecnologías de la educación}

Rama (2018) sostiene que la la virtualización de la educación es la mayor innovación de los procesos educativos a escala global de inicios del siglo XXI, y no cabe duda, más aún en el contexto actual. Se ha ingresado a una nueva fase de la alfabetización digital, 
la cual constituye un derecho de todo ciudadano moderno como sostiene López-Barajas (2009: pp. 30-31).

Los educadores, y en general la ciudadanía, han de saber que la alfabetización digital es un derecho del ciudadano en la sociedad moderna, y que hoy ésta adquiere nuevos matices y exigencias como son: el conocimiento como usuarios de las Nuevas Tecnologías de la Comunicación y la Información, como medio para incorporarse a la vida social y laboral. (López-Barajas, 2009: pp. 30-31)

En esta nueva escena mundial en la cual predomina un nuevo entorno educativo las tecnologías de la educación y la educación a distancia han tomado protagonismo. En las sociedades del conocimiento, hoy se consideran a las nuevas tecnologías de la comunicación y de la información como un medio para desarrollar la autonomía, la pluralidad y el respeto a los derechos humanos (UNESCO, 2005).

Hoy es una realidad palpable la superación de las fronteras en el mundo de la comunicación. Puesto que la enseñanza a distancia sigue siendo comunicación profesoralumno cuando media la separación espacio-temporal entre ambos, se refiere a los canales capaces de superarla. El canal más antiguo para ello es el texto escrito, erigido gracias a la imprenta en el primer "mass media", al que hoy cabe añadir la radio, teléfono, ordenador y todos los modernos sistemas de archivo de imagen y sonido, multimedia e internet.

Según el peso específico otorgado a cada medio surgirá un sistema u otro de enseñanza a distancia: el texto escrito da lugar a la tradicional enseñanza por correspondencia; las emisiones televisadas a la enseñanza por televisión, etc, y combinaciones que permite construir un sistema "multi-media" integrado. Las posibilidades de cada medio dan lugar a una potencialidad didáctica propia, que lo hace especialmente apropiado para determinado tipo de objetivos. Pero se puede afirmar que el medio maestro sigue siendo el medio impreso, y no sólo por motivos económicos. Mediante la lectura se penetra en el mundo de la abstracción de manera personalizada, puesto que es el propio sujeto quien marca el ritmo de aprendizaje, hora y lugar de lectura; por el contrario, los restantes medios conllevan un ritmo secuencial fijo y mayores condicionamientos, de tal modo que, a menos que aparezcan totalmente integrados en un sistema "multimedia”, muchas veces no son aprovechados por una gran parte del alumnado.

La educación a distancia como modalidad de la educación se articula sobre la base del incesante desarrollo de la tecnología de la información y comunicación, de nuevas relaciones sociopedagógicas entre docentes y alumnos, y de nuevas manifestaciones culturales. Bajo estas condiciones, la modalidad simultáneamente se renueva, enriquece, afronta nuevos retos, responsabilidades; por parte del que aprende. Estos factores no sólo dan contexto contemporáneo a la educación a distancia, sino que generan nuevas 
dinámicas socioeducativas que permiten entender la innovación de la modalidad, permitiendo su enriquecimiento y desarrollo continuo (Bazán, 2006, pp. 61).

En la educación a distancia se observa la búsqueda de correspondencia entre las relaciones socioeducativas, docentes y discentes, y el nivel de desarrollo alcanzado por las tecnologías de la información y la comunicación que circulan en el ámbito de la educación. Se busca equilibrar esta relación sobre la base de los fines y objetivos de la educación y de las aplicaciones multimedia. El equilibrio de reciprocidad en las relaciones sociopedagógicas estaría dado en la centralidad que ocupa el alumno en los procesos de enseñanza-aprendizaje, en esta modalidad con fines y objetivos bien definidos y con recursos didácticos adecuadamente seleccionados.

Con el desarrollo de la informática, la computadora se ha convertido en un multimedio en sí misma, al sintetizar en sus programas textos, imágenes, sonidos, animación etc. El computador, aplicado a la educación, es el artefacto que mejor simboliza las condiciones contemporáneas de la labor tanto docente como de aprendizaje. "Sin este artefacto es muy poco probable que se realicen acciones en la modalidad. El ordenador probablemente sea el artefacto que mejor representa simbólicamente la cultura contemporánea y las potencialidades de la educación a distancia, así como las facilidades para el aprendizaje como sostiene (Bazán, 2006, pp. 63).

En resumen, las tecnologías de la educación en sus múltiples presentaciones constituye una permanente motivación al estudiante que siempre está dispuesto a descubrir y potenciar lo que la tecnología y sus dispositivo como entornos nos brinda, por lo que que los medios sincrónicos y asincrónicos utilizados correctamente permitirán un óptimo proceso de enseñanza y aprendizaje.

\section{La Comunicación en el Sistema de Educación a Distancia}

La comunicación educador-educando no es resultado de la improvisación, ni sólo de naturaleza informal. La comunicación y sus cauces han de estar previstos en el organigrama funcional del sistema educativo, y en los calendarios y horarios de la planificación del curso. La comunicación ha de basarse en una información puntual de las múltiples variables que inciden en el proceso de enseñanza-aprendizaje (López-Barajas, 1988, pp. 62-63).

El núcleo de la comunicación educador-educando se configura hacia el conocimiento de las carencias y potencialidades del alumno, en primer lugar, y el autoconocimiento y aceptación del educando en un segundo momento. La comunicación para que sea educativa necesita caracterizarse como estímulo cualificado de mejora y autoexigencia. Las notas específicas de la comunicación educador-educando son: brevedad, claridad, cordialidad, adecuación, conveniencia y abierta a cualquier tema. La 
frecuencia excesiva o la falta de significado puede llevar al cansancio de comunicación a la pérdida de frescura e interés.

Las modalidades de comunicación según perspectivas distintas pueden ser individual y grupal; informal y orgánica; horizontal y vertical; ascendente y descendente; y oral o escrita. En la organización de un sistema participativo y autónomo deben caber todas las modalidades. Dependerá en cada caso del contenido de la comunicación. El cual ha de seleccionarse cuidadosamente, ha de tenerse presente a quien va dirigido, si reúne las notas anteriormente indicadas y el contexto específico, a fin de prever las reacciones individuales o de grupo.

El objetivo principal de los cauces de comunicación es facilitar la comunicación educador- educando para poder desarrollar la capacidad de expresión y comprensión de todos, la integración de esfuerzos y sacrificios en una misma dirección. Las líneas de comunicación formal forman la red de comunicaciones, la cual debe estar definida, expresada y conocida por todos los agentes de la educación en cada sistema específico. Las comunicaciones escritas, que presentan indudables ventajas respecto de la objetividad del mensaje, deben cuidarse especialmente, sobre todo las que se dirigen fuera del sistema institucional (autoridades académicas, padres etc.).

El Tutor o Profesor encargado de curso, que tuviese la función de atender personalmente a un grupo de alumnos, será el cauce ordinario de comunicación con los educandos. La periodicidad adecuada en la comunicación educador-educando deberá ser frecuente y versar no sólo sobre cuestiones alrededor de los cursos o temas escolares, sino también orientaciones para resolver dificultades de la vida familiar, profesional y personal.

Los canales de comunicación y los recursos en los que se apoyaba la educación a distancia en sus inicios mantenían ciertamente esa separación de espacio y tiempo, por lo que la interacción entre los agentes principales era sumamente limitada. Estos postulados veían incomprensible la posibilidad de educar con unos recursos y unos canales que no facilitaban la interacción, de ahí que, desde sus comienzos, la educación a distancia fuera considerada una enseńanza de segunda clase, ya que no era capaz de aportar la interacción y los elementos básicos propios de toda relación educativa. La enseñanza únicamente era posible si se desarrollaba en el mismo espacio y tiempo, cara a cara. A la vez, los únicos contextos propicios para llevar a cabo la educación eran las instituciones educativas formales y las aulas, en tanto que escenarios educativos enfocados para facilitar las interacciones físicas. Esta visión ha sido superada según García, Ruiz y Domínguez (2007) debido a tres factores interrelacionados artífices de un cambio de enfoque radical que son los siguientes:

- La afirmación de la educación como proceso a lo largo de la vida.

- La convicción de que todo espacio de interacción humana es un escenario educativo. 
- La consolidación de las tecnologías de información y comunicación como canal de comunicación y de recursos didácticos.

Lo notable de estos tres factores es que inciden en el cambio tanto en la enseñanza presencial como a distancia, ya que a partir de ellos se derriba la identificación de la educación con los espacios y tiempos socialmente determinados (colegios universidades, cursos etc.), ámbitos que durante siglos han organizado, de forma exclusiva y excluyente, toda propuesta de formación. Cuando se rompe esta convicción, se abre las posibilidades educativas insospechadas que van desde los entornos virtuales de aprendizaje, a otros entornos cotidianos como pueden ser los espacios de ocio o de trabajo.

La organización de la comunicación significativa se orienta hacia las necesidades e intereses del educando, hacia centros de interés y experiencia, y la configuración de un vínculo común entre disciplinas. La comunicación se ha de estructurar en todos homogéneos, alrededor de unidades con significación en sí mismas. La comunicación educador-educando implica un proceso educativo total, que es de naturaleza dual: asimilación-acomodación.

Existen diversos enfoques teóricos del aprendizaje que muestran la necesidad de modificar el papel del alumno en relación con los procesos de construcción del conocimiento, abandonando el lugar de receptores de información por el de protagonistas de dichos procesos, de manera individual y grupal. Esta concepción pedagógica, resulta imperiosa e ineludible en la educación a distancia y desde esta perspectiva el diseño del sistema debe realizarse en función del protagonismo del alumno, en un entorno que favorezca su aprendizaje.

Como se ha afirmado líneas arriba las propuestas de educación a distancia vienen mostrando grandes renovaciones no sólo en los medios utilizados sino también en el enriquecimiento de las relaciones establecidas entre los diferentes actores del proceso y de éstos con los materiales producidos. Han alentado estos cambios las actuales teorías de la enseñanza y el aprendizaje, por un lado, y por otro, las nuevas tecnologías capaces de proveer de herramientas que posibilitan el desarrollo de un proceso mediatizado, prometiendo dar respuesta a algunos problemas de comunicación existentes en el modelo clásico de educación a distancia.

La óptima comunicación del educador incide en el autoconcepto del educando es así como los educadores pueden verificar que tras el refuerzo positivo (la alabanza), el autoconcepto discente, su autoestima, aumenta en grado. Por el contrario, los efectos del reproche, la sanción sistemática, la configuración del autoconcepto disminuye, se hace de signo negativo. El ajuste personal, escolar y social de los alumnos se relaciona más significativamente con las características de la comunicación que con los resultados del aprendizaje, el rendimiento. La comunicación profesor-alumno hace que las calificaciones escolares puedan ser percibidas o no como referidas a la personalidad global 
del estudiante. Si la comunicación identifica n o solo carencias son potencialidades que todo educando tiene, diferenciando otros rasgos del desarrollo, el clima del aula es estimulante y positivo incluso para aquellos alumnos con dificultades de aprendizaje.

En la actualidad la tecnología electrónica transforma la relación educación y comunicación, el espacio vital que cada persona siente como propio, se ve influido desde fuera por factores y variables técnico-sociales que presentan valores diferentes. Es así que los sistemas educativos y sociales se encuentran inmersos en un desarrollo tecnológico acelerado que atraviesa todas sus dimensiones. La tecnología de la educación se configura como la respuesta que reoriente el sentido humano de la acción docente como así de la utilización de los procedimientos y de recursos, coherentes con la aplicación de los principios derivados de la teoría de la educación.

En el contexto del adelanto y el desarrollo resulta lógico el desarrollo de los sistemas educativos a distancia, donde las características de la comunicación educadoreducando ha de concebirse teniendo en cuenta estos conceptos: (Mena, Rodríguez y Díez, 2005, pp. 207-209)

- Distancia transaccional: se refiere a la distancia que existe en las relaciones educativas, determinada por la cantidad y calidad del diálogo que tiene lugar entre el estudiante y el profesor y la estructuración que existe en el diseño del curso. Cuando el curso está muy estructurado y el diálogo es mínimo se establece la mayor distancia transaccional. Esta situación puede darse en cualquier modalidad, por ejemplo, en un curso presencial no puede existir diálogo. Cuando el estudiante asume su control y como consecuencia de ello aumenta el diálogo con el profesor, la distancia transaccional se reduce. Lo importante no es dónde están o cómo se comunican los actores del proceso sino la calidad y cantidad de su interacción a través de los materiales e instancias de comunicación.

- Interacción: hace referencia a la acción recíproca entre sujetos, entre personas. Tiene que ver con la comunicación y la interrelación desarrollada entre los actores involucrados en la propuesta educativa, en función de la enseñanza y el aprendizaje. En este sentido, los procesos de cooperación, colaboración en la construcción del conocimiento es posible por la interacción. La mediación pedagógica explicitada en los materiales y en la práctica de los tutores debe orientarse a impulsar y sostener los procesos. Lo importante es de qué manera se estimula la interacción, cómo se saca el mejor provecho de la tecnología, cómo se maneja sus aspectos negativos y se supera los inconvenientes, tanto en una comunicación sincrónica como asincrónica.

- Interactividad: este proceso hace referencia a la acción recíproca, a la confrontación directa del estudiante con el material o dispositivo que viabiliza el contenido del aprendizaje. Se entiende por interactividad al hecho que ambos extremos del canal de comunicación participan emitiendo mensajes, que son 
recibidos e interpretados por el otro extremo, y que, de alguna manera, influyen en el modo como continúa desarrollándose el diálogo. Personas y maquinas participan en el juego de la interactividad con diferentes niveles de procesamiento cognitivo de la información y de capacidad de toma de decisiones, pero la esencia del proceso es siempre la misma: alguien emite un mensaje, otro lo recibe, procesa dicha información y emite una respuesta que es un nuevo mensaje.

Una buena interactividad se ve favorecida por:

- Diseńo didáctico y tecnológico adecuado

- Guía y orientación permanente

- Información necesaria y suficiente a disposición

- Buena conectividad

- Retroalimentación continua

Los criterios que se pueden utilizar para determinar la calidad de este proceso son:

- Número de veces que el alumno participa

- Rango de actividades en las que participa

- Relevancia de la participación

- Producción

Todos los recursos que se utilicen deben favorecer la interactividad, los materiales de hecho son una pieza clave para lograr el desarrollo de los procesos, por lo cual un modelo que plantee propuestas abiertas, motivadoras y participativas para la construcción del conocimiento en forma autónoma se transforma en una condición básica para favorecer el aprendizaje.

Los modelos de diseño didáctico constituyen una especie de puente o enlace entre las teorías del aprendizaje y las teorías sobre la enseñanza. El modelo de diseño que se adopte para elaborar los materiales del programa a distancia condicionará, en cierta medida, el aprendizaje que logren los alumnos ya que este determinará el tipo de actividades y las formas de interacción propuestas.

Diferentes teorías del aprendizaje caracterizan de diversas formas los ámbitos de interacción. En la teoría conductista se menciona por ejemplo de contextos instructivos, mientras que en las teorías constructivistas se habla de ambientes, entornos de aprendizaje, siendo sus propuestas más abiertas y flexibles, promoviendo la autorregulación personal y grupal de los procesos de aprendizaje, y la interacción para la toma de decisiones acerca de cómo se desarrollaran dichos procesos. 
Encontrar una respuesta al problema de investigación planteado ha llevado a realizar una revisión de las teorías de aprendizaje sobre todo aquellas que sostiene que el proceso educativo sea presencial o distancia tiene un elemento básico que es la comunicación, medio que permitirá que el estudiante logre aprendizajes significativos, ayudado por la acción facilitadora del docente y además por la existencia de un sistema de comunicación formal en proceso educativo a distancia.

\section{Estrategias educativas}

La efectividad de la enseñanza a distancia depende de la adecuada organización de los distintos elementos que en ella intervienen; el resultado ha de ser una enseñanza sistemática, planificada y racional, donde la improvisación ocupe una parte mínima. Si en la enseñanza presencial se puede remediar inmediatamente un error, en la enseñanza a distancia resulta poco menos que imposible. Por ello se hace más necesario aún una planificación tecnológica y el desarrollo d estrategias educativas

Puesto que las estrategias dependen de los objetivos y de los recursos disponibles, la elaboración de una estrategia concreta precisa de la clarificación previa de ambos extremos. Piénsese que no es lo mismo trazar una estrategia de enseñanza a distancia para informar a un colectivo sobre la adopción de medidas sanitarias, mediante la radio y televisión, que pretender actualizar un maestro de primaria mediante textos impresos.

Las tácticas y estrategias que a continuación se anuncian son de cariz genérico y referido a los objetivos indicados como válidos para cualquier programa de educación a distancia. Pretenden, eso sí, ser coherentes con los supuestos de tipo psicológico y social presentados. Para no hacer exhaustivo este apartado, los enunciados serán lo más escuetos posible:

La motivación inicial se logrará mantener siempre y cuando el programa responda a las expectativas despertadas. La enseñanza a distancia, en mayor medida que la presencial, ha de apoyarse en las necesidades reales de la población a la que va dirigida, de ahí que los sistemas de captación de alumnos deban indicar claramente la naturaleza del programa y sus exigencias, a fin de no despertar falsas expectativas, que luego no se podrán mantener.

Los medios didácticos escogidos han de responder al doble principio de eficacia y economía. Cabe, sin embargo, el empleo de medios más costosos (TV a color, filmes sonoros, impresión gráfica a color, etc.) con propósitos motivadores y para prestigiar socialmente al sistema. Desde la estricta perspectiva didáctica, el medio maestro sigue siendo el texto impreso (tal vez sustituible en un próximo futuro por el "video texto") puesto que resulta el más económico y funcional para el estudio, por otro lado, resulta insustituible para la consecución de ciertos objetivos abstractos y estético-literarios. La 
radio es el medio de comunicación más íntimo, que aporta el profundo poder motivador de la palabra oral; su participación en la educación a distancia es utilísima para marcar la secuencia del estudio, para dar informaciones inmediatas y como aplicación de conocimientos. El teléfono proporciona seguridad al estudiante; aun cuando no fuere muy utilizado, el solo hecho de tenerlo a su disposición es suficiente para despertar confianza hacia el sistema. La televisión, ordenadores, etc. tienen utilidad para la consecución de objetivos concretos, sin olvidar lo dicho sobre el prestigio social del sistema.

Todo estudiante que se inicia en el sistema de enseñanza a distancia ha de ser mínimamente informado y adiestrado para el estudio en solitario. Esta preparación no puede convertirse en algo puramente formal, sino que ha de estar directamente vinculada con el aprendizaje de contenidos interesantes para él. Los sistemas de enseñanza a distancia han de procurar vincularse a instituciones y programas de educación presencial, a fin de no quedar marginados de las líneas vanguardistas de investigación educativa. Tal vinculación contribuirá también al conocimiento objetivo de esta modalidad de enseñanza y, por tanto, podrá eliminar los prejuicios sociales que hoy aún tiene que soportar.

Desde esta perspectiva, el diseño y selección de las estrategias es sin duda relevante en todo proyecto de educación a distancia dado que a través de ellos se establecerán las comunicaciones. Para su selección es necesario seguir algunos criterios (Mena, Rodríguez y Diez (2005 pp. 167):

- Los distintos medios deben ser analizados a partir de sus condiciones concretas de fijación y, principalmente, por las funciones pedagógicas que puedan cumplir en relación con las necesidades de educación de una determinada población.

- Los distintos tipos de medios imponen ciertas características a los mensajes. Hay grandes diferencias entre trasmitir una información en un material impreso y difundirlas a través de la televisión a canal abierto. En cada caso, esa relación de los alumnos con los medios asume características distintas, lo que condiciona la propia estructura de los mensajes.

- En los medios audiovisuales se utilizan ciertos recursos como la inmediatez, la redundancia y la repetición, que no son tan apropiados para los medios impresos. Esto es así porque mientras los medios audiovisuales se distribuyen en el tiempo haciendo más lógica la reiteración, los impresos lo hacen en el espacio; al permanecer fijos y completos en el tiempo, los mensajes escritos hacen que la redundancia y la repetición sean consideradas tediosas cuando no incorrectas.

- Con relación a la forma en como las personas se relacionan con los distintos medios: la lectura de un texto exige el dominio de ciertas habilidades lectoras y el conocimiento de algunas normas de lectura predeterminadas; a su vez, permite interrupciones, retornos y relecturas en forma inmediata. Interactuar con una computadora implica otro tipo de dominio donde se exige no sólo alfabetización en lecto-escritura sino también conocimientos de informática. 
La variedad de medios tiene sus características intrínsecas, aspecto que debe tomarse en cuenta para una correcta elección. Según Bates. (1999. pp. 57-85) Se entiende como conversación entre el usuario y el sistema (o entre el usuario y el diseñador debemos tener unos criterios básicos al momento seleccionar: los cuales son cuatro: el acceso, costos, enseñanza aprendizaje e interactividad. Nos enfocaremos en los dos últimos:

- Enseñanza y aprendizaje: Hay tres cuestiones básicas de las funciones pedagógicas: ¿qué tipos de aprendizaje se pretenden lograr? ¿qué propuestas de enseñanza serán mejores promotoras de dichos aprendizajes? ¿cuáles son las mejores tecnologías para apoyar la enseñanza y aprendizaje?

- Interactividad y aceptación por parte de los sujetos: Un aspecto básico del uso de la tecnología en la educación y la capacitación es la "interfaz" entre personas y máquinas, a saber, la forma en que los estudiantes y los maestros pueden interactuar con una tecnología o mediante ella.

Existen tres elementos en la interfaz entre personas y máquinas: la necesidad de un aprendizaje activo, la necesidad de tecnologías adecuadas o sencillas y la necesidad de un control de maestros y estudiantes sobre las actividades de enseñanza y aprendizaje. Es necesario tener una respuesta para: ¿qué tipo de interacción implica esta tecnología? ¿qué grado de dificultad supone?

- Organización: La ejecución exitosa del proyecto, si bien requiere de la compra e instalación de equipos, la contratación de personal técnico la capacitación del personal docente, necesita también algunos cambios en la organización de la institución. De manera que en esta área deben de responderse las siguientes interrogantes: ¿cuáles son los requisitos de organización y las barreras que deben considerarse para poder emplear eficazmente esta tecnología? ¿qué cambios implica para la organización?

- Novedad: Según Bates es el criterio menos importante, pero a menudo resulta que una nueva tecnología crea mayores expectativas. Además permite considerar como síntoma de cambio y desarrollo de las instituciones. De considerar la respuesta al siguiente interrogante: ¿qué tan nueva es esta tecnología?

- Rapidez: En una sociedad sujeta a cambios rápidos, los cursos deben adaptarse con rapidez y actualizarse con facilidad. Es necesario responder a: ¿qué tan rápido pueden montarse los cursos con esta tecnología? ¿qué tan rápido pueden cambiarse los materiales?

Con las indicaciones y criterios expuestos lo que se pretende conseguir o evitar es la tendencia a repetir con las nuevas tecnologías lo que siempre se ha hecho sin ellas (Aparici, 2005). 
Por ejemplo, reproducir en la pantalla de la computadora el mismo texto que se encuentra en un manual impreso, el mismo mapa o dibujo, cuando se podría hacer un uso creativo de multimedia. Pero no sólo se trata de que se reproduzca los mismos ejemplos utilizados en otros medios, sino que también se repiten modelos comunicativos y concepciones reproductoras del proceso de enseñanza-aprendizaje. De poco sirve usar el correo electrónico, si vamos a hacer un uso convencional de este recurso.

Las actuales teorías de aprendizaje y de enseñanza están mostrando la necesidad de estructurar y organizar la información que llega al estudiante de acuerdo a sus necesidades y características de este, proporcionando un mayor grado de participación y apli-cación de sus estrategias cognitivas. Por ejemplo, organizando los contenidos a partir de situaciones integradoras, globales, de temas o prácticas, en el marco de la materia, disciplina o campo profesional al que pertenece.

Esta metodología guarda relación con principios de la Teoría Constructivista del aprendizaje, alejándose del enfoque clásico (conductista), usado tradicionalmente en la educación a distancia, que se caracteriza por presentar la información en pequeñas unidades.

Finalmente, tratándose de comunicación a distancia la motivación resulta aún más necesaria, de modo que aparece como requisito inicial. La preocupación del sistema didáctico estribará precisamente en su mantenimiento. Todos los recursos que se utilicen deben favorecer la interactividad, los materiales de hecho son una pieza clave para lograr el desarrollo de los procesos, por lo cual un modelo que plantee propuestas abiertas, motivadoras y participativas para la construcción del conocimiento en forma autónoma se transforma en una condición básica para favorecer el aprendizaje.

\section{A modo de conclusiones}

La modalidad de educación a distancia tiene, en la idea de sociedad de la información, un potencial enorme tanto para impartir enseńanza como para asistir a la propuesta de un nuevo y cualitativo tipo de aprendizaje. Como modalidad de la educación se articula sobre la base del incesante desarrollo de la tecnología de la información y comunicación, de nuevas relaciones sociopedagógicas entre docentes y alumnos, y de nuevas manifestaciones culturales. Bajo estas condiciones, la modalidad simultáneamente se renueva, enriquece, afronta nuevos retos, responsabilidades; por parte del que aprende. Estos factores no sólo dan contexto contemporáneo a la educación a distancia, sino que generan nuevas dinámicas socioeducativas que permiten entender la innovación de la modalidad, permitiendo su enriquecimiento y desarrollo continuo.

En todo sistema educativo es eminentemente un proceso de comunicación donde la información incluye de modo conexo el contenido y la manera o modalidad de trans- 
mitir el contenido. Es por ello por lo que el sistema educativo, en este caso a distancia no solo está obligado a estructurarse de acuerdo con los objetivos que persigue sino también de acuerdo con las legalidades del aprendizaje humano.

Sea el nombre que adopte en el sistema docente o docente/tutor la comunicación que establece en el proceso no es producto de la improvisación, mucho menos de la informalidad, ésta ha de ser prevista en el organigrama funcional del sistema educativo y en los calendarios y horarios de la planificación del curso o programa educativo. Las estrategias de comunicación educativa en esta modalidad deben permitir una comunicación personalizada entre docente/ tutor y el alumno que lo conlleve a orientar, dirigir y supervisar el proceso de enseñanza-aprendizaje de éstos según sus necesidades particulares.

El desarrollo de la capacidad del trabajo autónomo tiene que ser la estrategia en la que se sustenta toda acción docente en la formación no presencial, sin esta capacidad se hace difícil el seguimiento de un curso de esta forma, podríamos decir que es la condición sine qua non que debe tener un estudiante no presencial. El desarrollo de esta capacidad se ejercita, en gran parte, a través de pautas que reciben el nombre de guías de aprendizaje, ejercicios, lecturas etc. Y el resultado de esta conciencia de aprendizaje, es sin duda, el elemento más motivador en los procesos de formación no presencial. Lo esencial es que se promueva la autonomía en el estudiante para que gradualmente vaya adquiriendo hábitos de estudio independientes y resolución de problemas con mayor autonomía.

\section{Referencias}

Aparici, R. (1997). "Mitos de la educación a distancia y las nuevas tecnologías", en Rodríguez, M. y Ahijado M. (1999), la educación a distancia en tiempos de cambios: Nuevas generaciones, viejos conflictos. Madrid: De la Torre

Bates, A.W.(1999). La Tecnología en la Enseñanza Abierta y la Educación a distancia. México, Editorial Trillas, 334pp.

Bazán, J. (2006). El Protagonismo del estudiante en la educación a distancia. Lima, Fondo Editorial de la Facultad de Ciencias Sociales de la UNMSM. 104 pp.

García, L. Ruiz, M. y Domínguez D. (2007). De la educación a distancia a la Educación Virtual. Barcelona, Editorial Ariel. 303 pp.

López-Barajas Z. (1988). Comunicación educador-educando (presencial y a distancia). Barcelona, Compilado por J. Sarramona, pp. 65-79

López-Barajas E. (2009) Alfabetización virtual y gestión del conocimiento En Revista Teoría de la Educación. Educación y Cultura en La Sociedad de la Información. TESI, 10 (2), 2009, 02-429 Vol. 10. No2. Julio Universidad Nacional de Educación a Distancia 
Mena, M. Rodríguez, 1. y Diez, M. (2005). El diseño de proyectos de educación a distancia. Buenos Aires, Editorial Stella. 318pp.

Rama, Claudio (2018), Políticas, tensiones y tendencias de la educación a distancia y virtual en América Latina, Salta, EUCASA

Organización de las Naciones Unidas para la Educación, la Ciencia y la Cultura (UNESCO). (2005) Hacia las sociedades del conocimiento (Informe publicado en el 2005). Descargado de http:// unesdoc.unesco.org/images/ 0014/001419/141908s.pdf 